\title{
Generalized Multiuser Orthogonal Space-Division Multiplexing
}

\author{
Zhengang Pan, Student Member, IEEE, Kai-Kit Wong, Member, IEEE, and Tung-Sang Ng, Fellow, IEEE
}

\begin{abstract}
This paper addresses the problem of performing orthogonal space-division multiplexing (OSDM) for downlink, point-to-multipoint communications when multiple antennas are utilized at the base station (BS) and (optionally) all mobile stations (MS). Based on a closed-form antenna weight solution for single-user multiple-input multiple-output communications in the presence of other receiver points, we devise an iterative algorithm that finds the multiuser antenna weights for OSDM in downlink or broadcast channels. Upon convergence, each mobile user will receive only the desired activated spatial modes with no cochannel interference. Necessary and sufficient conditions for the existence of OSDM among the number of mobile users, the number of transmit antennas at the $\mathrm{BS}$, and the number of receive antennas at the MS, are also derived. The assumption for the proposed method is that the BS knows the channels for all MS's and that the channel dynamics are quasi-stationary.
\end{abstract}

Index Terms-Broadcast channels, cochannel interference, diversity, joint-channel diagonalization, multiple-input multiple-output (MIMO), multiuser communications, orthogonal space-division multiplexing, smart antennas.

\section{INTRODUCTION}

Since mobile users are generally situated at different locations, they intrinsically occupy different physical channels in space. Signals at the same frequency band and time slot should therefore be differentiable by their spatial signatures. In uplink [from many mobile stations (MSs) to one base station (BS)], this can be done using multiuser detection [1], [2] or array signal processing for space-division multiple access (SDMA) [3], [4]. Nevertheless, reusing the same radio channel in downlink/broadcast channels (from one BS to many MSs) is a more challenging task because coordination among different MSs is not allowed.

Promoting spectral resource reuse in broadcast channels traces back several decades [5] and the method is based on so-called dirty-paper coding (DPC). By means of known interference precancellation at the transmitter, DPC encodes the data in a way that the codes align themselves as much as possible with each other so as to maximize the sum-capacity of a broadcast channel [6]-[9]. However, dirty-paper techniques are largely information-theoretic and worse, the encoding process to achieve the sum-capacity is data dependent. In other words, the cancellation needs to be done independently for every symbol.

Manuscript received February 17, 2003; revised November 8, 2003; accepted November 10, 2003. The editor coordinating the review of this paper and approving it for publication is I. B. Collings. This work was supported in part by the Hong Kong Research Grant Council (HKU 7175/03E) and the University of Hong Kong Research Committee.

The authors are with the Department of Electrical and Electronic Engineering, University of Hong Kong, Hong Kong, China (e-mail: kitwong @ieee.org).

Digital Object Identifier 10.1109/TWC.2004.837449
In this paper, we investigate a practical technique that aims to fully utilize the rich diversity existing in broadcast channels for performance improvements. In particular, we address the problem of performing orthogonal space-division multiplexing (OSDM) for a wireless system in downlink where multiple antennas are utilized at the BS and (optionally) all MSs. Solutions to this problem can be viewed as one form of generalized beamforming that includes any signal processing of antenna array even if no beam pattern is formed in the case where the direction-of-arrival may be ill defined because of uncorrelated scattering. Unlike DPC, by jointly optimizing the BS and MS antennas, we project the multiuser signals onto orthogonal subspaces so that the interference seen by all the MSs is eliminated (or controlled) according to their spatial signatures.

With multiuser multiple-input multiple-output (MIMO) antennas, schemes to obtain OSDM in broadcast channels have been proposed by several researchers [10]-[14]. In [10]-[12], the broadcast channel is made block diagonal so that cochannel interference (CCI) is eliminated by placing nulls at the antennas of all the unintended MSs. Since receiver combining was not considered together with the optimization, these methods fail to obtain the rich diversity of the channels and require unnecessarily larger number of antennas at the BS. More recently in [13] and [14], Wong et al. proposed an iterative method that jointly updates the BS and MS antennas for downlink OSDM. However, the scheme in [13] suffers from several shortcomings. First, the way the antenna weights are obtained requires one additional antenna for interference space at every MS. Besides, when the number of BS antennas is greater than the number of cochannel signals existing in the system, the algorithm automatically disables the additional BS antennas, which results in considerable performance degradation.

Our work is different in that we devise an independent approach that iteratively optimizes the BS and MS antenna weights to perform OSDM in broadcast channels. Unlike [10]-[12], our method places the nulls at the combined signal outputs of the unintended MS. In so doing, diversity can be preserved while ensuring there is no interference on the activated spatial modes. Most importantly, it will be shown by simulation to have significant performance gain when compared to the previously proposed methods [10]-[14]. In addition, we shall derive the necessary and sufficient conditions on the number of BS and MS antennas given the number of activated spatial modes of the users. The proposed method assumes that the BS knows the channel state information (CSI) for all MSs.

This paper is organized as follows. In Section II, we introduce the multiuser MIMO system model. Section III formulates the optimization problem and presents an iterative algorithm for downlink OSDM. In Section IV, the necessary and sufficient conditions for OSDM to exist are derived. Simulation results are given in Section V. We conclude this paper in Section VI. 


\section{MUlTiUSER MIMO SySTEM MODEL}

For an $M$-user MIMO system where $n_{T}$ antennas are located at the BS and $n_{R, m}$ antennas are located at the $m$ th MS, at each symbol duration, multiple spatial modes, denoted by $N_{m}$, can be supported for the $m$ th user. Denoting $z_{n}^{(m)}$ as the symbol sent on the $n$th spatial mode by the $m$ th user (the time index is omitted for conciseness), we can write $\mathbf{z}_{m}=\left[\begin{array}{llll}z_{1}^{(m)} & z_{2}^{(m)} & \cdots & z_{N_{m}}^{(m)}\end{array}\right]^{T}$ as the symbol vector for user $m$ where the superscript $T$ denotes the transposition. The overall system can be written as [13]

$$
\hat{\mathbf{z}}_{m}=\mathbf{R}_{m}^{\dagger}\left(\mathbf{H}_{m} \sum_{m^{\prime}=1}^{M} \mathbf{T}_{m^{\prime}} \mathbf{z}_{m^{\prime}}+\mathbf{n}_{m}\right) \quad \forall m
$$

where $\mathbf{z}_{m^{\prime}} \in \mathbb{C}^{N_{m^{\prime}}}$ denotes the symbol vector transmitted from the $m^{\prime}$ th user, $\mathbf{T}_{m^{\prime}} \in \mathbb{C}^{n_{T} \times N_{m^{\prime}}}$ denotes the linear transmitter processing for the $m^{\prime}$ th user's symbols, $\mathbf{H}_{m} \in \mathbb{C}^{n_{R, m} \times n_{T}}$ is the channel matrix from the BS to the $m$ th MS, and $\mathbf{R}_{m} \in$ $\mathbb{C}^{n_{R, m} \times N_{m}}$ denotes the linear receiver processing for the $m$ th user's symbols, and $\mathbf{n}_{m} \in \mathbb{C}^{n_{R, m}}$ is the noise vector at MS $m$, whose entries are zero-mean complex Gaussian random variables with variance of $N_{0} / 2$ per dimension. Likewise, $\hat{\mathbf{z}}_{m} \in$ $\mathbb{C}^{N_{m}}$ is the $m$ th user signal vector that contains the soft-output estimates of the transmitted symbols, and the superscript $\dagger$ represents conjugate transposition.

We also find it useful to define the multiuser transmit weight matrix as $\mathbf{T}=\left[\begin{array}{llll}\mathbf{T}_{1} & \mathbf{T}_{2} & \cdots & \mathbf{T}_{M}\end{array}\right]$ and the multiuser transmitted symbol vector as $\mathbf{z}=\left[\begin{array}{llll}\mathbf{z}_{1}^{T} & \mathbf{z}_{2}^{T} & \cdots & \mathbf{z}_{M}^{T}\end{array}\right]^{T}$. Consequently, (1) becomes

$$
\hat{\mathbf{z}}_{m}=\mathbf{R}_{m}^{\dagger}\left(\mathbf{H}_{m} \mathbf{T z}+\mathbf{n}_{m}\right) \quad \forall m
$$

\section{DOWNLINK OSDM}

Our objective is to obtain a multiuser channel diagonalization in downlink by finding the joint antenna weight matrices $\mathbf{T}, \mathbf{R}_{1}, \ldots, \mathbf{R}_{M}$. Presumably, if OSDM exists, it will generally have more than one (typically infinitely many) solution. Among these solutions, we aim to choose the one that optimizes the performance of the multiuser MIMO system. Mathematically, this can be written as

$$
\left(\mathbf{T}, \mathbf{R}_{1}, \ldots, \mathbf{R}_{M}\right)_{\text {opt }}=\arg \max _{\mathbf{T}, \mathbf{R}_{1}, \ldots, \mathbf{R}_{M}} \sum_{m=1}^{M}\left\|\boldsymbol{\Lambda}_{m}\right\|^{2}
$$

where $\|\cdot\|$ denotes the Frobenius norm of the input matrix [16] and $\boldsymbol{\Lambda}_{m}$ is defined by

$$
\begin{aligned}
& \mathbf{R}_{m}^{\dagger} \mathbf{H}_{m} \mathbf{T} \\
& =\left[\begin{array}{lll}
\mathbf{0}_{1} \cdots \mathbf{0}_{m-1} & \underbrace{\boldsymbol{\Lambda}_{m}}_{m \text { th subblock matrix }} & \left.\mathbf{0}_{m+1} \cdots \mathbf{0}_{M}\right] \forall m
\end{array}\right]
\end{aligned}
$$

where

$$
\boldsymbol{\Lambda}_{m}=\operatorname{diag}\left(\lambda_{1}^{(m)}, \lambda_{2}^{(m)}, \ldots, \lambda_{N_{m}}^{(m)}\right)
$$

is of dimension $N_{m} \times N_{m}$ and $\lambda_{n}^{(m)}$ corresponds to the resultant channel gain for the $n$th spatial mode (or the $n$th signal stream) of the $m$ th MS. Likewise, $\mathbf{0}_{m^{\prime}}$ is the $m^{\prime}$ th subblock zero matrix of dimension $N_{m} \times N_{m^{\prime}}$. From definition (4), each subblock matrix corresponds to the CCI from each user to MS $m$. The optimization will be performed by setting $\left\|\mathbf{t}_{n}^{(m)}\right\|=1 \forall m, n$.

We approach this problem by first finding the optimal transmit and receive weight matrices $\mathbf{T}_{m}$ and $\mathbf{R}_{m}$ for a particular user $m$, given the receive weight matrices for other users $\left\{\mathbf{R}_{\tilde{m}}\right\}_{\tilde{m} \neq m}$. The weight matrices for user $m$ are found in order that the resultant channel gains at MS $m$ are maximized and the transmit weights cause no CCI to all other MSs' outputs. Then the weight matrices for other users are updated one after another until convergence.

Define an equivalent multiuser channel matrix $\mathbf{H}_{e}$ as

$$
\mathbf{H}_{e} \triangleq\left[\begin{array}{c}
\mathbf{R}_{1}^{\dagger} \mathbf{H}_{1} \\
\vdots \\
\mathbf{R}_{M}^{\dagger} \mathbf{H}_{M}
\end{array}\right] \in \mathbb{C}^{\left(\sum_{m=1}^{M} N_{m}\right) \times n_{T}} .
$$

Assuming that the receive weight matrices, $\mathbf{R}_{1}, \ldots, \mathbf{R}_{m-1}$, $\mathbf{R}_{m+1}, \ldots, \mathbf{R}_{M}$, are fixed and known at the BS, the BS aims to find the best $\mathbf{T}_{m}$ and $\mathbf{R}_{m}$ that optimize the performance of the $m$ th user link while causing no CCI at the other MS so that

$$
\left(\mathbf{T}_{m}, \mathbf{R}_{m}\right)_{\mathrm{opt}}=\arg \max _{\mathbf{T}_{m}, \mathbf{R}_{m}}\left\|\boldsymbol{\Lambda}_{m}\right\|^{2}
$$

and

$$
\mathbf{H}_{e} \mathbf{T}_{m}=\left[\begin{array}{c}
\mathbf{0}_{1}^{T} \\
\vdots \\
\mathbf{0}_{m-1}^{T} \\
\Lambda_{m}^{T} \\
\mathbf{0}_{m+1}^{T} \\
\vdots \\
\mathbf{0}_{M}
\end{array}\right] \in \mathbb{C}\left(\sum_{m^{\prime}=1}^{M} N_{m^{\prime}}\right) \times N_{m}
$$

To accomplish (7) and (8), we define a LACK- $m$ matrix $\mathbf{H}_{e}^{(m)-}$ as

$$
\mathbf{H}_{e}^{(m)-\triangleq}\left[\begin{array}{c}
\mathbf{R}_{1}^{\dagger} \mathbf{H}_{1} \\
\vdots \\
\mathbf{R}_{m-1}^{\dagger} \mathbf{H}_{m-1} \\
\mathbf{R}_{m+1}^{\dagger} \mathbf{H}_{m+1} \\
\vdots \\
\mathbf{R}_{M}^{\dagger} \mathbf{H}_{M}
\end{array}\right] \in \mathbb{C}\left(\sum_{\substack{m^{\prime}=1 \\
m^{\prime} \neq m}}^{M} N_{m^{\prime}}\right) \times n_{T}
$$

Note that the LACK- $m$ matrix agrees with $\mathbf{H}_{e}$ except that the block $\mathbf{R}_{m}^{\dagger} \mathbf{H}_{m}$ is missing. Obviously, in order to satisfy the nullification condition (8), we must have

$$
\mathbf{T}_{m} \in \operatorname{null}\left\{\mathbf{H}_{e}^{(m)-}\right\}
$$

where null $\{\cdot\}$ denotes the nullspace of the input matrix. By letting the orthonormal basis of $\operatorname{null}\left\{\mathbf{H}_{e}^{(m)-}\right\}$ be 
$\mathbf{Q}_{m}=\left[\mathbf{q}_{1}^{(m)} \mathbf{q}_{2}^{(m)} \ldots\right]$, we can write $\mathbf{T}_{m}=\mathbf{Q}_{m} \mathbf{B}_{m}$, where $\mathbf{B}_{m}$ denotes the coordinate transformation under the basis $\mathbf{Q}_{m}$. The remaining task is then to choose $\mathbf{B}_{m}$ such that

$$
\left(\mathbf{B}_{m}, \mathbf{R}_{m}\right)_{\mathrm{opt}}=\arg \max _{\mathbf{B}_{m}, \mathbf{R}_{m}}\left\|\boldsymbol{\Lambda}_{m}\right\|^{2}
$$

and

$$
\mathbf{R}_{m}^{\dagger} \mathbf{H}_{m} \mathbf{Q}_{m} \mathbf{B}_{m}=\boldsymbol{\Lambda}_{m}
$$

Because of (10), it automatically ensures interference-free operation at all the signal outputs of the other MS. Accordingly, the problem is now reduced to finding the optimal processing $\mathbf{B}_{m}$ and $\mathbf{R}_{m}$ of a single-user MIMO system supporting multiple spatial eigenmodes.

It has been shown in [15] that singular value decomposition (SVD) can be taken as the optimal solution in achieving the capacity for a single-user MIMO link. Therefore, $\mathbf{R}_{m}$ and $\mathbf{B}_{m}$ can be updated by

$$
\left(\mathbf{R}_{m}\right)_{\mathrm{opt}}=\left.\mathbf{U}_{m}\right|_{1 \leftrightarrow N_{m}}
$$

and

$$
\left(\mathbf{B}_{m}\right)_{\mathrm{opt}}=\left.\mathbf{V}_{m}\right|_{1 \leftrightarrow N_{m}}
$$

in which $\mathbf{U}_{m}\left(\mathbf{V}_{m}\right)$ is the left (right) singular matrix of $\mathbf{H}_{m} \mathbf{Q}_{m}$ (i.e., $\mathbf{H}_{m} \mathbf{Q}_{m}=\mathbf{U}_{m} \boldsymbol{\Lambda}_{m} \mathbf{V}_{m}^{\dagger}$ ) and the notation $\left.\cdot\right|_{1 \leftrightarrow N_{m}}$ collects the column vectors that correspond to the $N_{m}$ largest singular values. As a result, the optimal transmit weight matrix can be found as

$$
\left(\mathbf{T}_{m}\right)_{\mathrm{opt}}=\mathbf{Q}_{m}\left(\mathbf{B}_{m}\right)_{\mathrm{opt}} .
$$

Using the solution stated above, an iterative algorithm is formulated as follows.

1) Initialize $\mathbf{R}_{m}=\mathbf{I} \forall m$ where $\mathbf{I}$ is an identity matrix of appropriate dimensions.

2) For each $m$, form the matrix $\mathbf{H}_{e}^{(m)-}$ using (9), and obtain the orthonormal basis of null $\left\{\mathbf{H}_{e}^{(m)-}\right\}, \mathbf{Q}_{m}$. By calculating the SVD of $\mathbf{H}_{m} \mathbf{Q}_{m}$, obtain the optimal solution of $\left(\mathbf{T}_{m}\right)_{\text {opt }}$ and $\left(\mathbf{R}_{m}\right)_{\text {opt }}$ using (15) and (13), respectively.

3) Compute $\epsilon=\operatorname{off}\left(\mathbf{H}_{e} \mathbf{T}\right)$ where

$$
\mathrm{off}(\mathbf{A}) \triangleq \sum_{k, \ell}\left|a_{k, \ell}\right|^{2}
$$

in which $|\cdot|$ takes the modulus of a complex number. If $\epsilon \leq$ $\epsilon_{T h}\left(=10^{-12}\right.$ typically), proceed to Step 4); otherwise, go back to Step 2).

4) The convergence is said to be achieved and normalization is then performed for all columns of $\mathbf{T}$ to satisfy the power constraint.

Numerical results have demonstrated that the proposed algorithm can achieve OSDM (4) whenever the conditions provided in the next section are satisfied. Throughout this paper, we shall refer to the above algorithm as an iterative nullspace-directed SVD (iterative Nu-SVD).

\section{NECESSARY AND SUFFICIENT CONDITIONS FOR OSDM}

Theorem 4.1-The Number of BS Antennas (17): For an $M$-user OSDM system with user $m$ transmitting on $N_{m}$ spatial modes, the number of BS antennas $n_{T}$ must be greater than or equal to the total number of activated spatial modes (or signal streams) within the system, i.e.,

$$
n_{T} \geq \sum_{m=1}^{M} N_{m} .
$$

Proof: The constraint (4) can be rewritten as

$$
\mathbf{H}_{e} \mathbf{T}=\left[\begin{array}{ccc}
\boldsymbol{\Lambda}_{1} & \mathbf{0} & \cdots \\
\mathbf{0} & \boldsymbol{\Lambda}_{2} & \\
\vdots & & \ddots
\end{array}\right] \in \mathbb{C}_{m=1}^{M} N_{m} \times \sum_{m=1}^{M} N_{m} .
$$

It follows from the rank property that

$$
\begin{aligned}
\sum_{m=1}^{M} N_{m} & =\operatorname{rank}\left\{\mathbf{H}_{e} \mathbf{T}\right\} \\
& \leq \operatorname{rank}\{\mathbf{T}\}\left(\operatorname{or} \operatorname{rank}\left\{\mathbf{H}_{e}\right\}\right) \\
& \leq \min \left(n_{T}, \sum_{m=1}^{M} N_{m}\right) .
\end{aligned}
$$

As such, to make (4) possible, (17) must be satisfied.

Theorem 4.2-The Number of MS Antennas (20): For a mobile user of an OSDM system to support $N_{m}$ spatial modes, the number of MS antennas $n_{R_{m}}$ must be greater than or equal to the number of activated spatial modes for that MS, i.e.,

$$
n_{R_{m}} \geq N_{m} \quad \forall m .
$$

Proof: According to (4), we have $\operatorname{rank}\left\{\mathbf{H}_{m}\right\} \geq$ $\operatorname{rank}\left\{\mathbf{R}_{m}^{\dagger} \mathbf{H}_{m} \mathbf{T}\right\}=N_{m}$. Since $\mathbf{H}_{m}$ is of size $n_{T} \times n_{R_{m}}$, $\min \left(n_{T}, n_{R_{m}}\right) \geq N_{m}$. From Theorem 4.1, $n_{T} \geq N_{m}$ and (20) follow.

Theorem 4.3-Sufficient Conditions for the Minimum Number of BS and MS Antennas (17) and (20): If both (17) and (20) are satisfied, it is always sufficient to achieve OSDM among and within users (4).

Proof: To complete the proof, we here provide a straightforward solution that can achieve OSDM under Theorems 4.1 and 4.2. To start with, we define a multiuser channel matrix $\mathbf{H}_{0}$ as

$$
\mathbf{H}_{0} \triangleq\left[\begin{array}{c}
\mathbf{H}_{1} \\
\mathbf{H}_{2} \\
\vdots
\end{array}\right] \in \mathbb{C}\left(\sum_{m=1}^{M} n_{R_{m} \times n_{T}}\right)
$$

and assume that any $\min \left(n_{T}, \sum_{m} n_{R_{m}}\right)$ rows of the matrix $\mathbf{H}_{0}$ are uncorrelated. Hence, $\mathbf{H}_{0}$ has full rank. [Here, it means $\operatorname{rank}\left\{\mathbf{H}_{0}\right\}=\min \left(n_{T}, \sum_{m} n_{R_{m}}\right)$ [16].] This assumption is reasonable because the fading coefficients from different antenna pairs are generally independent.

Based on Theoream 4.2 (i.e., $n_{R_{m}} \geq N_{m}$ ), we set

$$
\mathbf{R}_{m}=\left[\begin{array}{c}
\mathbf{I}_{N_{m}} \\
\mathbf{0}
\end{array}\right] \in \mathbb{C}^{n_{R_{m}} \times N_{m}}
$$




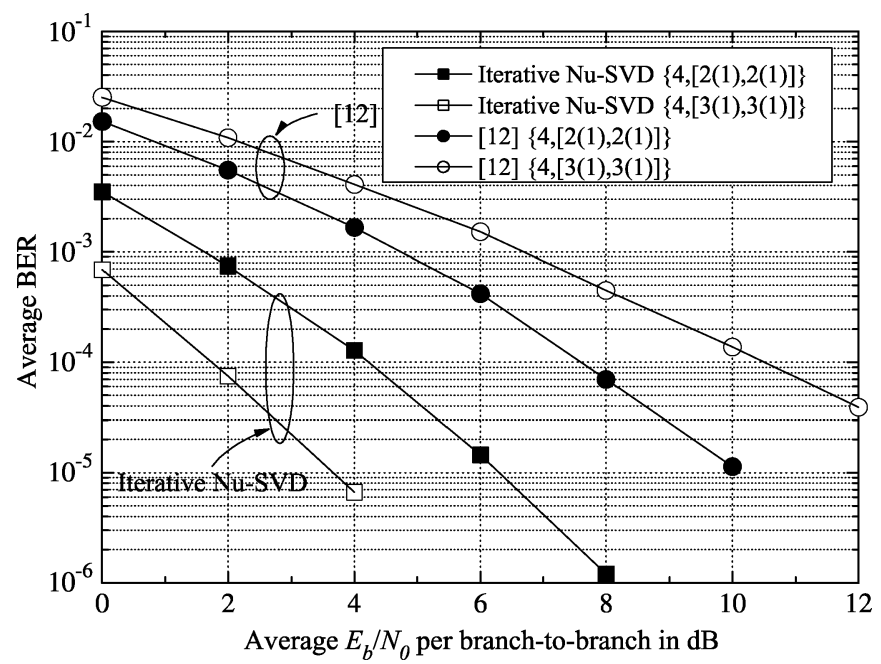

Fig. 1. Performance comparison of iterative Nu-SVD and the method in [12] for a multiuser MIMO antenna system with $N_{m}=1 \forall m$.

where $\mathbf{I}_{N_{m}}$ is an $N_{m} \times N_{m}$ identity matrix and $\mathbf{0}$ is an $\left(n_{R_{m}}-\right.$ $\left.N_{m}\right) \times N_{m}$ zero matrix. Then, we can compute $\mathbf{H}_{e}$ using (6). It gives

$$
\mathbf{H}_{e}=\left[\begin{array}{c}
\left.\mathbf{H}_{1}\right|_{1 \leftrightarrow N_{1}} \\
\left.\mathbf{H}_{2}\right|_{1 \leftrightarrow N_{2}} \\
\vdots \\
\left.\mathbf{H}_{M}\right|_{1 \leftrightarrow N_{m}}
\end{array}\right] \in \mathbb{C}\left(\sum_{m=1}^{M} N_{m} \times n_{T}\right) .
$$

From Theorem 4.1 (i.e., $n_{T} \geq \sum_{m} N_{m}$ ) and the full rank assumption of $\mathbf{H}_{0}, \mathbf{H}_{e}$ has full row rank. Therefore, there exists a matrix, given by $\mathbf{T}=\mathbf{H}_{e}^{\dagger}\left(\mathbf{H}_{e} \mathbf{H}_{e}^{\dagger}\right)^{-1}$, which is the pseudoinverse of $\mathbf{H}_{e}$ [16] so that $\mathbf{H}_{e} \mathbf{T}=\mathbf{I}$ satisfying (4). This completes the proof.

An observation here is that the required number of BS antennas depends only on the number of coexisted spatial modes in the system, but not the number of antennas at the MS as required in [10]-[12]. To make a comparison, consider a five-user system with $n_{R, m}=3$ and $N_{m}=1 \forall m$. In theory, it needs only five BS antennas while the systems in [10]-[12] require 13 BS antennas.

\section{Simulation Results}

Simulation results on the average bit error probability for various signal-to-noise ratio (SNR) (defined as the average $E_{b} / N_{0}$ per branch-to-branch) will be given to assess the performance of the proposed system. The channel model used in the simulations is a quasi-static flat Rayleigh fading channel. Four quadrature amplitude modulation (4-QAM) is assumed. For convenience, we use the notation $\left\{n_{T},\left[n_{R_{1}}\left(N_{1}\right), \ldots, n_{R_{M}}\left(N_{M}\right)\right]\right\}$ to denote an $M$-user MIMO system that has $n_{T}$ BS antennas and MS $m$ has $n_{R_{m}}$ antennas to support $N_{m}$ spatial streams.

Fig. 1 provides the average BER results of the proposed iterative $\mathrm{Nu}-\mathrm{SVD}$ and the approach in [12] for $\{4,[2(1), 2(1)]\}$ and $\{4,[3(1), 3(1)]\}$ systems. As can be seen, the performance of iterative Nu-SVD is significantly better than that of [12]. Most importantly, for the method in [12], the performance gets worse if the number of MS antennas increases. This is not true for our proposed method because for iterative Nu-SVD, only

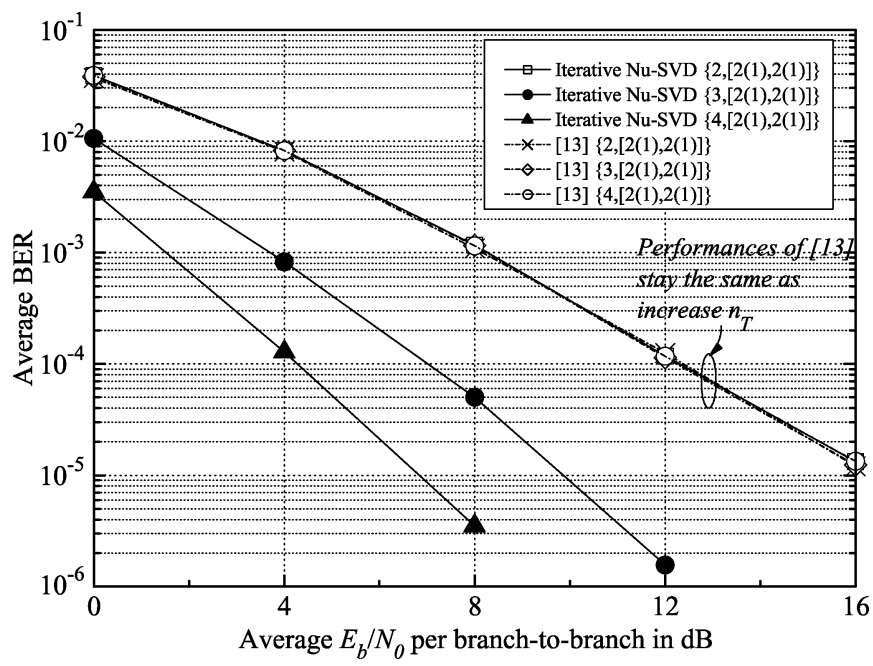

Fig. 2. Performance comparison of iterative Nu-SVD and the method in [13] for a multiuser MIMO antenna system with $N_{m}=1 \forall m$.

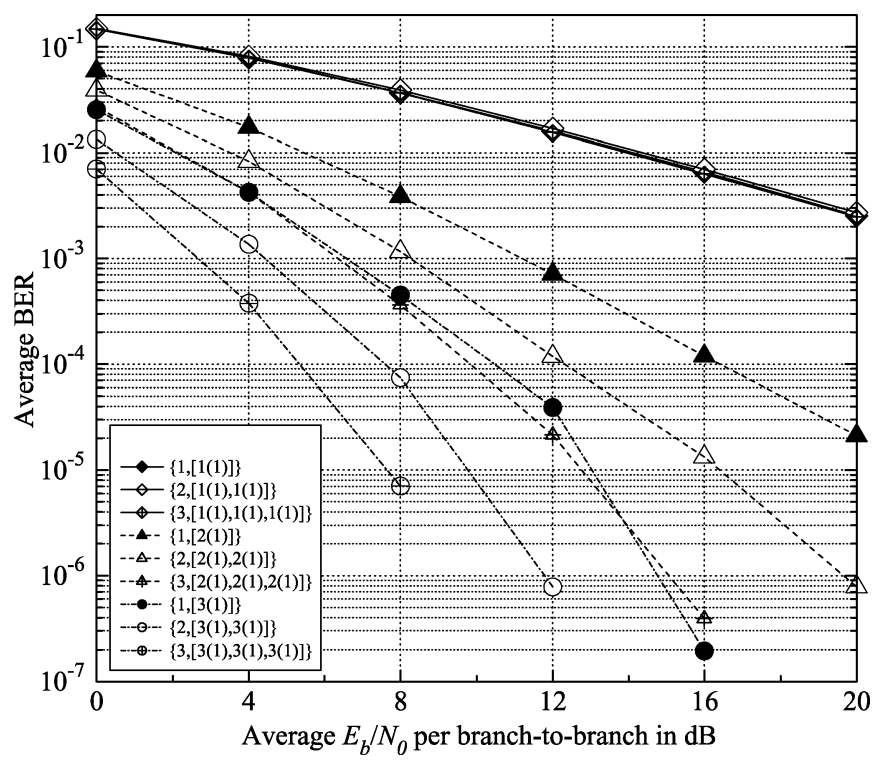

Fig. 3. Performance comparison of multiuser MIMO systems with various number of receive antennas and $N_{m}=1 \forall m$.

$N_{m}(=1)$ degrees of freedom are needed at the BS for CCI suppression while the method in [12] requires $n_{R}(=2 o r 3)$ degrees of freedom. The remaining degrees of freedom left at the BS can be utilized for diversity enhancement.

In Fig. 2, we present the results of the iterative Nu-SVD and the method in [13] for $\left\{n_{T},[2(1), 2(1)]\right\}$ systems by varying the number of BS antennas $n_{T}$. Results reveal that the performance of [13] is limited by the total number of spatial modes in the system. By contrast, the performance of the proposed method improves as the number of BS antennas goes beyond the number of spatial modes. About an order of magnitude reduction in BER is achieved by the iterative Nu-SVD for every addition of BS antennas when compared to the method in [13]. The change in the slopes of the curves indicates the enhanced diversity of the systems.

Fig. 3 shows the improved performance of the iterative $\mathrm{Nu}-\mathrm{SVD}$ by increasing the number of MS antennas (from one to three). In this figure, we set $n_{T}=M$, and each user 


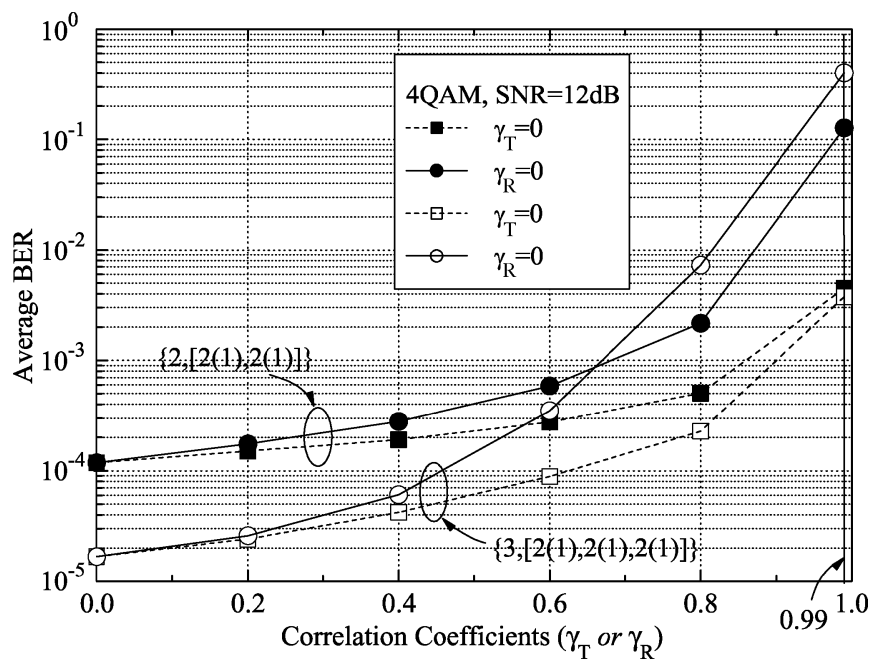

Fig. 4. Average BER performance of iterative Nu-SVD with various spatial correlation coefficients $\gamma_{T}$ and $\gamma_{R}$.

is supporting one spatial mode (i.e., $N_{m}=1 \forall m$ ). Results show that the performances of $\{1,[1(1)]\},\{2,[1(1), 1(1)]\}$ and $\{3,[1(1), 1(1), 1(1)]\}$ are the same, meaning that the iterative $\mathrm{Nu}-\mathrm{SVD}$ can manage the cochannel signals effectively in transmit mode and a multiuser system appears virtually to be three uncoupled single-user systems. Furthermore, as the number of MS antennas increases, the BER performance improves accordingly because of the receiver antenna diversity at the MS. More intriguingly, for a given number of antennas per MS, more diversity is obtained for a system with more users. This is evidenced by noting that the performance of $\{2,[2(1), 2(1)]\}$ is better than that of $\{1,[2(1)]\}$. This is because with more users in the system, more BS antennas are used and the increase in the degree of freedom contributes partly to maintain the orthogonalization and partly to obtain diversity.

We conclude this section by providing results in the presence of spatial correlation between the antennas at the BS and MS. To do this, we use the correlation channel model in [17] for $\mathbf{H}_{0}$ in (21) so that the correlation can be characterized by the transmit and receive correlation coefficients $\gamma_{T}$ and $\gamma_{R}$. In Fig. 4, we plot the results of $\{2,[2(1), 2(1)]\}$ and $\{3,[2(1), 2(1), 2(1)]\}$ systems for various values of $\gamma_{T}$ and $\gamma_{R}$ when $\mathrm{SNR}=12 \mathrm{~dB}$. Clearly, the system performance degrades as the spatial correlation increases. Surprisingly, near-perfect performance can be obtained with spatial correlation as high as 0.4 . Also, note that the performance is more sensitive to the spatial correlation at the BS than at the MS. This can be explained by recognizing that as $\gamma_{T}$ increases, not only does it reduce the diversity achievable at the MS but also the signals for different MSs become more difficult to separate. Results in this figure also indicate that systems with more users are more sensitive to the spatial correlation.

\section{CONCLUSIONS}

This letter has proposed an approach (iterative Nu-SVD) for OSDM in broadcast channels. Necessary and sufficient conditions for OSDM also have been derived. Simulation results have shown that iterative Nu-SVD significantly outperforms the OSDM approaches previously published in [10]-[13] and requires the minimal number of BS and MS antennas for OSDM.

\section{REFERENCES}

[1] T. Van Etten, "Maximum likelihood receiver for multiple channel transmission systems," IEEE Trans. Commun., vol. COM-24, pp. 276-283, Feb. 1976.

[2] S. Verdú, "Minimum probability of error for asynchronous Gaussian multiple-access channels," IEEE Trans. Inform. Theory, vol. 32, no. IT-1, pp. 85-96, Jan. 1986.

[3] J. H. Winters, J. Salz, and R. D. Gitlin, "The impact of antenna diversity on the capacity of wireless communication systems," IEEE Trans. Commun., vol. 42, pp. 1740-1751, 1994.

[4] B. Suard, G. H. Xu, H. Liu, and T. Kailath, "Uplink channel capacity of space-division-multiple-access schemes," IEEE Trans. Inform. Theory, vol. 44, pp. 1468-1476, July 1998.

[5] M. Costa, "Writing on dirty paper," IEEE Trans. Inform. Theory, vol. IT-29, pp. 439-441, May 1983.

[6] A. J. Goldsmith and M. Effros, "The capacity region of broadcast channels with intersymbol interference and colored Gaussian noise," IEEE Trans. Inform. Theory, vol. 47, pp. 211-219, Jan. 2001.

[7] D. Tse and P. Viswanath, "On the capacity of the multiple antenna broadcast channel," in Proc. DIMACS Series Discrete Math. Theoretical Computer Science, 2003.

[8] G. J. Foschini and A. H. Diaz, "Dirty paper coding: perturbing off the infinite dimensional lattice limit," in Proc. DIMACS Series Discrete Math. Theoretical Computer Science, 2003.

[9] W. Yu and J. M. Cioffi, "Sum capacity of a Gaussian vector broadcast channel,", submitted for publication.

[10] R. L. U. Choi and R. D. Murch, "A transmit pre-processing technique for multiuser MIMO systems: a decomposition approach," IEEE Trans. Wireless Commun., vol. 3, pp. 20-24, Jan. 2003.

[11] M. Rim, "Multiuser downlink beamforming with multiple transmit and receive antennas," Electron. Lett., vol. 38, pp. 1725-1726, Dec. 2002.

[12] Q. H. Spencer and M. Haardt, "Capacity and downlink transmission algorithms for a multiuser MIMO channel," in Proc. 36th Asilomar Conf. Signals, Systems, Computers, Pacific Grove, CA, Nov. 2002.

[13] K. K. Wong, R. D. Murch, and K. Ben Letaief, "A joint-channel diagonalization for multiuser MIMO antenna systems," IEEE Trans. Wireless Commun., vol. 2, pp. 773-786, July 2003.

[14] K. K. Wong, "Performance analysis of single and multi-user MIMO diversity channels using Nakagami- $m$ distribution," IEEE Trans. Wireless Commun., vol. 3, pp. 1043-1047, July 2004.

[15] G. G. Raleigh and J. M. Cioffi, "Spatio-temporal coding for wireless communications," IEEE Trans. Commun., vol. 46, pp. 357-366, Mar. 1998.

[16] G. H. Golub and C. F. Van Loan, Matrix Computation, 2nd ed. Baltimore, MD: The Johns Hopkins Univ. Press.

[17] D. Chizhik, J. Ling, P. W. Wolniansky, R. A. Valenzuela, N. Costa, and K. Huber, "Multiple-input-multiple-output measurements and modeling in Manhattan," IEEE J. Select. Areas Commun., vol. 21, pp. 321-331, Apr. 2003. 